\title{
Design and Simulation of Intelligent Controller of Cleaning Robot
}

\author{
Li Fangyun, Zhao Wei \\ Institute of Technology, East China Jiaotong University, Nanchang Jiangxi, 330100, China
}

Keywords: cleaning robot; controller; design; simulation

\begin{abstract}
Cleaning robot is widely applied in modern society. This paper introduces the development of cleaning robot and its utilization characteristics and requirements, and briefly analyzes the design and simulation of cleaning robot controller from various aspects.

With the development of computer technology and artificial intelligence, intelligent robot is no stranger to modern people. Intelligent robot, with high operational efficiency, helps a lot in various aspects in human life. Therefore, the development of cleaning robot becomes a trend of the times.
\end{abstract}

\section{Introduction to Cleaning Robot}

In fact, the concept of "cleaning robot" was put forward long ago. In 1920, Czech writer K. Kebike first used the concept of "robot" in his sci-fi works. Subsequently, various types of robots have appeared widely in various science fiction novels. Today, with the mature development of computer technology, establishment of corresponding production line, the growth in the living standard, and price production of computer-related products, cleaning robot has been widely used in life and has become a prospected future, and the application scope of China's cleaning robot is relatively positive.

Cleaning robot provides cleaning services. Generally, there are some requirements for cleaning robot. First of all, the cleaning function. There are different types of cleaning, and each type has its set cleaning function. Secondly, the utilization safety. Thirdly, high performance. Cleaning robot with high performance can ensure better operational status in complex environment to intelligently satisfy the demand of human. Fourthly, low interference. Low interference requires cleaning robot to have less impact on human life, such as less noise. And general cleaning robot shall meet these requirements.

\section{The Design of Controller Model}

Intelligent controller of cleaning robot is divided into route planning, fault avoidance, garbage absorption, environmental monitoring, which is described in detail.

\subsection{Route planning}

Route planning is an important aspect of cleaning robot controller. First of all, it can better accomplish the cleaning. In the operation, it shall reach designated area as much as possible, and the area where robot cannot reach is an important index to evaluate the cleaning function of the robot. In actual design, to enhance the cleaning function of robot, operation time of robot will be increased. It is not advisable to implement carpet-cleaning, which has high cleaning performance, whereas, the time required is too much. Therefore, the designer shall provide a certain constraint of algorithm to realize the controlling of performance.

\subsection{Fault avoidance}

Good fault avoidance is necessary for cleaning robot controller. In cleaning, cleaning robot will hit the wall, the corner of the table and something like inevitably, so it has to just the collision rapidly and make new route planning. Therefore, fault avoidance include the collision judgment and new route planning. Collision judgment include the judgment before and after the collision. The judgment after the collision means that after collision, the movement speed and direction will 
change to some extent, and programmer just need to make judgment according to the dynamic monitoring of movement speed and direction of cleaning robot. The judgment before the collision means that cleaning robot probes surrounding environment in movement, analyzes the data of surrounding environment through sensor, judges the fault in movement path and changes the path in time, which can effectively avoid the collision with cleaning robot. In general, fault analysis before the collision has high requirements for the performance of cleaning robot. In fault avoidance, the designer is required to optimize further the response time and accurate judgment of controller to ensure the better performance of cleaning robot.

\subsection{Garbage absorption}

Garbage adsorption requires the cleaning robot to mobilize the garbage adsorption function module and carry out the garbage adsorption during the movement. Garbage adsorption usually consists of two types, and the first is undifferentiated garbage adsorption, which refers to the behavior of continuous adsorption of garbage during the movement of the cleaning robot. The second one is judgmental garbage adsorption, which means that the cleaning robot judges the garbage on the path and adsorbs it during the movement. Each of these two methods has certain advantages and disadvantages. The second method is usually used more frequently without considering energy conservation and environmental protection issues.

\subsection{Environmental monitoring}

Different types of cleaning robots use different environmental sensors, including infrared sensors, temperature sensors, pressure sensors, etc. In general, in order to complete the specific functions of the cleaning robot, the designer will combine several different sensors to achieve a fixed function monitoring effect.

\section{Design and Simulation}

Before the creation and application of cleaning robot, relevant personnel shall conduct the design and simulation of virtual environment on it, which is described from seven aspects.

\subsection{Modelling}

There are a lot of virtual modeling software on the market, and users can choose the software according to their own needs. This design uses Open GL as the modeling software to carry out the corresponding modeling design of the cleaning robot. Open GL is a 3D modeling software with excellent performance. Its management functions and environment design functions can help designers quickly build realistic 3D model. It is worth noting that the 3D modeling of Open GL is relatively simple. Users can quickly understand the modeling work through 3D/2D conversion. Users can also directly conduct numerical design on model, which can better realize the rotation and translation of the model, which can save time in modeling.

\subsection{Movement simulation}

Movement is an important part of cleaning robot. The use of modern kinematics can better simulate the movement of cleaning robots. First, use the kinematics knowledge to analyze the motion trajectory of the robot. On this basis, a set of kinematic positive solution equations and kinematic inverse equations can be obtained. Second, the designer needs to design or use some existing procedures to analyze the kinematics positive solution equations, the kinematic inverse equations, and the connections between the two. In analysis, if the results obtained by the two equations are not consistent, this indicates that there are certain problems in the movement simulation of the cleaning robot, thus, the designer is required to analyze the cause of the problem, and then correct it with the kinematic design method after finding the cause of the problem.

The D-H parameter method is a common kinematic equation construction method, proposed by Denavit and Hartenberg in 1855 and has developed greatly in the current environment. The construction of the kinematic equation by the $\mathrm{D}-\mathrm{H}$ parameter method mainly includes three steps, 
the establishment of coordinates, the transformation of coordinates, and the realization of the equation.

\subsection{Collision detection}

Collision detection in a virtual environment is also referred to as contact detection and interference detection. Collision detection is divided into two parts, collision detection occurrence and collision position detection. With the development of computer technology, the technology related to collision detection has also been greatly developed. There are already some mature collision detection algorithms on the market, including ICollideGJK, SWIFT++, Vclip, etc.

Two methods for quickly detecting collisions are the distance tracking method and the bounding volume hierarchy method. The core idea of distance tracking method is to use the continuity of the motion model in time and geometry, and the nearest distance of the object in the adjacent motion process can be obtained by calculation. The core idea of bounding volume hierarchy method refers to the use of strict hierarchical bounding volume to form a hierarchical structure.

In collision detection, relevant personnel need to pay attention to several aspects of detection. First, 3D collision detection is required. Second, collision information can be accurately acquired and recorded, including collision contact points, collision distances, and penetration distances. Third, the system has high stability.

\subsection{Path generation}

The movement path of the cleaning robot is a very important part of the cleaning robot controller. The path is very much related to the cleaning performance of the cleaning robot. Therefore, the design and generation of path is very important. The path design of cleaning robots on the current market is to a large extent an imitation of human behavior. The designer needs to design several basic movements in the design process, and the cleaning robot needs to choose the motion path suitable for itself according to the actual situation and the algorithm.

\subsection{Simulation analysis with different friction coefficients}

Cleaning robots need to adapt to complex environments, so motion analysis on floors with different coefficients of friction is a very important part of cleaning robots. The designer needs to adjust the movement coefficient and the motion structure of the cleaning robot according to the motion of the cleaning robot on the floor with different friction coefficients, so that it can adapt to more complicated environments.

In simulation, the designer needs to conduct research on two aspects, the relationship between the movement speed of the cleaning robot and the friction coefficient, and the simulation of the relationship between the acceleration of the robot and friction coefficient. Considering the speed and acceleration under different friction coefficients is because the cleaning effect of traditional cleaning robot is largely related to the operational speed and operational acceleration of the cleaning robot.

On this basis, the designer needs to design two variables. The first one is the setting of friction coefficient. When setting the friction coefficient, the designer needs to ensure that the friction coefficient of the design is representative, which means that the coefficient of friction should be common in real life. For instance, the friction coefficient of $0.01,0.06,0.2$, and 0.3 have strong representativeness, the span of these four values is larger, and they are also more common in actual life. The second one is the design of motion type. The simulation needs to analyze the motion of the cleaning robot in different modes. The two motion modes of this simulation analysis are straight and steering.

In order to perform the simulation better, the designer also needs to make other settings, including setting the gravity parameter, which should use the surface gravity parameter, ie: $\mathrm{g}=9.8 \mathrm{~N} / \mathrm{kg}$. In addition, the design of the model quality is also very important, and the quality parameter used in this design is $30 \mathrm{~kg}$.

Through simulation design analysis, the results obtained in this study are that the friction coefficient has a great influence on the movement speed and motion acceleration of the cleaning 
robot. Therefore, the designer can design several different cleaning modes for the cleaning robot so that it can be adapted to cleaning with different friction coefficients.

\subsection{Simulation analysis of absorption effect}

The cleaning principle of cleaning robots is varied, and cleaning with absorption capacity is a common cleaning method. Therefore, simulation analysis of adsorption effect is also very important. In simulation analysis of adsorption effect, the designer needs to make three settings. First, the setting of the operational speed of the cleaning robot. The operational speed of the cleaning robot needs to match the actual environment, and meet the objective utilization conditions of the cleaning robot. Second, the setting of the adsorbate. In different environments, the cleaning effect that the cleaning robot needs to accomplish is different. For example, in a home environment, the main adsorbent of a cleaning robot is dust, impurities, and some other small adsorbate in the home. In factory environment, the adsorption of the cleaning robot has changed a lot, related to the production of the factory. The designer needs to make a suitable setting according to the application place of the cleaning robot. Most of the adsorbates selected in this study are small adsorbates. Third, the setting of absorption capacity. The absorption capacity of cleaning robots is related to the objective level of cleaning robots. In setting, the designer should not make the absorption and the actual situation very different. In general, designers can design several sets of different absorption capacities, analyze them in different environments and analyze the absorption effect of different absorption capacities, and design the absorption capacity suitable for the actual environment.

\subsection{The system stability of cleaning robot}

The system stability of the cleaning robot is also an aspect that needs to be evaluated. In general, there are three methods for analyzing the system stability of cleaning robots. The first one is source code analysis. Source code analysis refers to the analysis of the design source code of the cleaning robot controller. By analyzing the perfectness of the source code and other aspects, the designer can have a certain initial judgment on the stability of the cleaning robot controller. Second, the stability of the cleaning robot controller is analyzed with the functions provided by the simulation software. Third, virtual scenario simulation is performed to analyze the stability of the cleaning robot controller.

In general, the criteria for the stability of the cleaning robot controller include the three aspects. First, the specified functions can be completed according to a preset procedure, which means that the cleaning robot can complete the cleaning work within the range according to its preset program during the operation. Second, there is no program stuck or crash. In operation, if the amount of calculation is large, there will be problems in the system design (for example, the "deadlock phenomenon" on the program), the cleaning robot will be stuck or even a crash occur, which affects the performance of the cleaning robot. Third, there are no major system vulnerabilities. It is necessary to ensure that the operation of the cleaning robot is in the operational design, and an undesigned operating state shall not be generated, which is also called an incomplete operational state.

\section{Conclusion}

With the development of computer technology and artificial intelligence technology, cleaning robot is more widely used in human life. In the design and analysis of cleaning robot controller, designers are required to design in line planning, fault avoidance, garbage absorption, and environmental monitoring. Therefore, the designer can perform further simulation design on cleaning robot based on simulation methods in this paper.

\section{Acknowledgement}

Fund project: This paper comes from science and technology project of Jiangxi Education Department. Project Name: Design and Simulation of Intelligent Controller of Cleaning Robot. 
Project No. 161564

\section{References}

[1] Yan Zhengguo, Lv Guoqiang, Wei Meili. Design of Intelligent Motion Controller [J]. Industrial Control Computer, 2017, 30 (10): 51-52.

[2] Tang Wenzhen, Qian Ruiming. Design of a Glass Curtain Wall Cleaning Robot [J]. Machine Design and Manufacturing Engineering, 2017, 46 (1): 45-48.

[3] Yang Cunzhi, He Gang, Yao Lihui et al. Design of Self-propelled Barn Cleaning Robot [J]. Journal of Agricultural Mechanization Research, 2017, 39 (5): 90-94.

[4] Fang Xiangming, Fang Ming, Liu Tianyuan, et al. Research on Robot Simulation Design Based on Virtual Reality Technology [J]. Journal of Changchun University of Science and Technology (Natural Science Edition), 2016 (1): 61-65. 